Pacific Journal of Mathematics

AUTOMATIC CONTINUITY OF DERIVATIONS AND 


\title{
AUTOMATIC CONTINUITY OF DERIVATIONS AND EPIMORPHISMS
}

\author{
VOLKER RUNDE
}

\begin{abstract}
Two automatic continuity problems for derivations on commutative Banach algebras are discussed: (a) Every derivation on a commutative Banach algebra maps into the radical, and: (b) Derivations on semiprime Banach algebras are continuous. It is shown that (b) implies (a). Further, (b) is reduced to a smaller class of Banach algebras and proved in some special cases. Related results for epimorphisms are given.
\end{abstract}

1. Introduction. A derivation on an algebra $A$ is a linear mapping $D: A \rightarrow A$ that satisfies

$$
D(a b)=a D b+(D a) b \quad(a, b \in A) .
$$

In this paper we are concerned with derivations on commutative Banach algebras.

In 1955 Singer and Wermer ([10]) proved the now classical theorem: Every continuous derivation on a commutative Banach algebra maps into the radical. In the same paper they conjectured: (a) Every derivation on a commutative Banach algebra maps into the radical. In this context, it is reasonable to look for classes of Banach algebras on which every derivation is continuous. It was shown by B. E. Johnson that every derivation on a commutative, semisimple Banach algebra is continuous ([5]). Among the commutative Banach algebras the semisimple ones are characterized as those having no topologically nilpotent element other than zero; all known examples of discontinuous derivations on commutative Banach algebras depend crucially on the existence of nontrivial nilpotent elements in the algebraic sense ([7]). Thus, we might possibly generalize Johnson's theorem as follows: (b) Every derivation on a semiprime Banach algebra, i.e. on a commutative Banach algebra without nontrivial nilpotent elements, is continuous.

One result of this paper will be that (b) implies (a). Recently, (a) has been proved by Marc P. Thomas ([11]), whereas it still seems to be unknown whether (b) is true or not. 
I would like to thank Professor Dr. George Maltese, who recommended this topic to me, Dr. Michael Pannenberg for many interesting discussions, and particularly Dr. Jörg Eschmeier for his patient and helpful support.

2. Preliminaries. The following lemma is due to Garimella ([4]).

Lemma 1. Let $A$ be a commutative algebra, $P \subset A$ a prime ideal, and $D: A \rightarrow A$ a derivation. Then

$$
\left\{a \in P \mid D^{k} a \in P \text { for all } k \in \mathbb{N}\right\}
$$

is a prime ideal, too.

Hence, minimal prime ideals in commutative algebras are invariant under derivations. Let $A$ be a commutative algebra and $P \subset A$ a minimal prime ideal. Then every derivation $D: A \rightarrow A$ "drops" to a derivation $\widehat{D}: A / P \rightarrow A / P$ by

$$
\widehat{D}(a+P)=D a+P \quad(a \in A) .
$$

The algebra $A / P$ is an integral domain and may be easier to treat than $A$. However, in the case that $A$ is a Banach algebra, $A / P$ need not be a Banach algebra, for prime ideals are not necessarily closed.

Recall the concept of the separating space of a linear operator: Let $X, Y$ be Banach spaces, and $T: X \rightarrow Y$ a linear mapping. Then

$$
\begin{array}{r}
\mathscr{S}(T)=\left\{y \in Y \mid \text { there is a sequence }\left\{x_{k}\right\} \text { in } X \text { with } x_{k} \rightarrow 0,\right. \\
\text { and } \left.T x_{k} \rightarrow y\right\}
\end{array}
$$

is said to be the separating space of $T$; it is a closed, linear subspace of $Y$. By the closed graph theorem, $\mathscr{S}(T)$ is zero if and only if $T$ is continuous. Let $A$ be a Banach algebra. Then the separating space of a derivation on $A$ or of an epimorphism from another Banach algebra onto $A$ is a two-sided ideal $S \subset A$ with the following stability property: For each sequence $\left\{a_{k}\right\}_{k \geq 1}$ in $A$ there is an $n \in \mathbb{N}$ such that

$$
\overline{a_{1} \cdots a_{n} S}=\overline{a_{1} \cdots a_{k} S} \quad(k \geq n) .
$$

An arbitrary closed, two-sided ideal $S$ of $A$ which satisfies (2) is called a separating ideal ([2]).

The next lemma has been proved first in [2] for noncommutative Banach algebras; a simpler proof in the commutative case can be found in [1]. 
LEMma 2. Let $A$ be a commutative Banach algebra, $S \subset A$ a separating ideal, and $P \subset A$ a minimal prime ideal which does not contain $S$. Then $P$ is closed.

3. Automatic continuity of derivations on semiprime Banach algebras: a first characterization. The first theorem is analogous with an older result by Julian Cusack ([2]) for epimorphisms.

THEOREM 1. The following three statements are equivalent.

(i) Every derivation on a commutative Banach algebra has a nilpotent separating space.

(ii) Every derivation on a semiprime Banach algebra is continuous.

(iii) Every derivation on a Banach algebra which is an integral domain is continuous.

Proof. The only thing we have to show is that (iii) implies (i). We prove it by contraposition. Let $A$ be a commutative Banach algebra and $D: A \rightarrow A$ a derivation with nonnilpotent separating space. Then there is a minimal prime ideal $P \subset A$ that does not contain $\mathscr{S}(D)$. By Lemma 2, it is closed, and $A / P$ is a Banach algebra which is an integral domain. Then $\widehat{D}: A / P \rightarrow A / P$ defined according to (1) is a discontinuous derivation on $A / P$.

The equivalence of (ii) and (iii) has already been observed by Garimella ([4]). In the last section of this paper we shall add a fourth equivalent statement to Theorem 1.

4. Reducing the Singer-Wermer problem. It is a well-known theorem by B. E. Johnson that to prove the Singer-Wermer conjecture we may limit our attention to derivations on local Banach algebras, i.e. on commutative, unital Banach algebras possessing exactly one maximal ideal; this reduction of the problem is required in [11]. We shall reduce the problem a bit further.

THEOREM 2. The following three statements are equivalent.

(i) Every derivation on a commutative Banach algebra maps into the radical.

(ii) Every derivation on a local Banach algebra maps into the radical.

(iii) Every derivation on a local Banach algebra which is an integral domain maps into the radical.

Proof. The equivalence of (i) and (ii) is proved in [5]; for a polished proof see [8]. Assume that (iii) holds. Let $A$ be a local Banach algebra, 
$D: A \rightarrow A$ a derivation, and $P \subset A$ a minimal prime ideal. As $A$ is local, we have $P \subset \bar{P} \subset \operatorname{rad}(A)$. Let us first consider the case that $P$ contains $\mathscr{S}(D)$. In this case we regard the Banach algebra $A / \bar{P}$; let $\pi: A \rightarrow A / \bar{P}$ be the canonical epimorphism. By well-known properties of separating spaces $([9])$,

$$
\mathscr{S}(\pi \circ D)=\overline{\pi \mathscr{S}(D)}=\{0\},
$$

and $\pi \circ D$ is continuous. As $P$ is invariant under $D$, and $\pi \circ D$ is continuous, we have $(\pi \circ D)(\bar{P})=\{0\}$, and therefore, $D \bar{P} \subset \bar{P}$. So, we may define a continuous derivation $\widehat{D}$ on $A / \bar{P}$ by

$$
\widehat{D}(a+\bar{P})=D a+\bar{P} \quad(a \in A) .
$$

The Singer-Wermer theorem yields

$$
\widehat{D}(A / \bar{P}) \subset \operatorname{rad}(A / \bar{P})=\operatorname{rad}(A) / \bar{P},
$$

and hence, $D A \subset \operatorname{rad}(A)$. Now the second case: $\mathscr{S}(D) \not \subset P$. Then $P$ is closed by Lemma 2 , and $D$ drops as in (1) to a derivation $\widehat{D}$ on the local Banach algebra $A / P$ which is an integral domain. The assumption implies

$$
\widehat{D}(A / P) \subset \operatorname{rad}(A / P)=\operatorname{rad}(A) / P,
$$

and hence, $D A \subset \operatorname{rad}(A)$.

Some details of [11] will be simpler, if we assume the Banach algebra in question to be a local integral domain. Furthermore, Theorem 2 reveals a link between (a) and (b): If all derivations on semiprime Banach algebras were continuous, then, of course, all derivations on local Banach algebras which are integral domains would map into the radical. So, solving the automatic continuity problem for derivations on semiprime Banach algebras might yield new evidence of the SingerWermer conjecture.

5. Accessible and inaccessible prime ideals. Accessible prime ideals were introduced by Curtis in [1] to give a sufficient condition for the Singer-Wermer conjecture to hold.

Definition 1. Let $A$ be a commutative Banach algebra. A closed prime ideal $P \subset A$ will be called accessible, if it is the intersection of all closed ideals of $A$ properly containing it. Otherwise, $P$ is said to be inaccessible.

The following lemma due to Curtis displays the crucial property of accessible prime ideals. 
LEMMA 3. Let $A$ be a commutative Banach algebra, and $P \subset A$ an accessible prime ideal. Then $P$ contains every separating ideal of $A$.

5.1. Some automatic continuity theorems. Let $A$ be a Banach algebra which is an integral domain with accessible zero-ideal. It is a trivial conclusion from Lemma 3 that every derivation on $A$ and every epimorphism onto $A$ is continuous. However, the accessibility of the zero-ideal is often difficult to verify directly. In this subsection we shall give sufficient conditions for the zero-ideal of an integral domain to be accessible.

LEMMA 4. Let $A$ be a Banach algebra which is an integral domain, let $I \subset A$ be a closed ideal, and let there be an element $z \in A$ such that $\bigcap_{k=1}^{\infty} z^{k} I=\{0\}$. Then $\{0\}$ is accessible.

Proof. Let $H$ denote the intersection of all nonzero, closed ideals of $A$. Assume $H$ to be nonzero. Then, $\overline{z H}=H$ holds, and by the Mittag-Leffler theorem (see [3]), $\overline{\bigcap_{k=1}^{\infty} z^{k} H}=H$. In particular, $\bigcap_{k=1}^{\infty} z^{k} H \neq\{0\}$. As $I$ is closed, we have $H \subset I$, and hence, $\bigcap_{k=1}^{\infty} z^{k} H \subset \bigcap_{k=1}^{\infty} z^{k} I=\{0\}$, which is a contradiction.

TheOREM 3. Let $A$ be a Banach algebra which is an integral domain, let $I \subset A$ be a closed ideal, and let there be an element $z \in A$ such that $\bigcap_{k=1}^{\infty} z^{k} I=\{0\}$. Then every derivation on $A$ and every epimorphism onto $A$ is continuous. In particular, all Banach algebra norms for $A$ are equivalent.

A similar, slightly weaker result only for derivations has been given by Garimella ([4]).

Now let $A$ be a Banach algebra which is a subalgebra of $\mathbb{C}[[X]]$, and assume that $A$ is not isomorphic to $\mathbb{C}$. Consider the linear functional $p_{0}: A \rightarrow \mathbb{C}$ that maps each power series in $A$ to its constant term. Then $p_{0}$ is either the zero-mapping or an epimorphism onto $\mathbb{C}$. In any case, there is a nonzero $f \in A$ for which the constant term vanishes; hence, $\bigcap_{k=1}^{\infty} f^{k} A=\{0\}$. We have thus proved:

Corollary 1. Let $A$ be a Banach algebra which is a subalgebra of $\mathbb{C}[[X]]$. Then every derivation on $A$ and every epimorphism onto $A$ is continuous. In particular, all Banach algebra norms for $A$ are equivalent.

Corollary 1 partly improves an older result by R. J. Loy ([6]): Let $A$ be a topological algebra which is a subalgebra of $\mathbb{C}[[X]]$, and which 
is a Fréchet space such that (i) the coordinate functionals $p_{k}: A \rightarrow \mathbb{C}$ $(k \in \mathbb{N})$ are continuous, and (ii) there is a sequence $\left\{\omega_{k}\right\}$ of strictly positive reals such that the family $\left\{\omega_{k} p_{k}\right\}$ is equicontinuous. Then every derivation on $A$ is continuous. In the Banach algebra case, (ii) is clearly superfluous. By Corollary 1 , we see that in this case we may omit (i), too.

Lemma 5. Let $A \neq \mathbb{C}$ be a Banach algebra which is an integral domain such that all prime ideals of $A$ are closed. Then $\{0\}$ is accessible.

Proof. Let $H$ denote again the intersection of all nonzero, closed ideals of $A$, and assume that there is a nonzero element $z \in H$. Then $\bigcap_{k=1}^{\infty} z^{k} H$ is dense in $H$. Consider the set $\left\{z^{k} \mid k \geq 1\right\}$; it is multiplicatively closed, and its intersection with $\bigcap_{k=1}^{\infty} z^{k} H$ is empty. Therefore, there is a closed prime ideal $P \subset A$ with $\bigcap_{k=1}^{\infty} z^{k} H \subset P$ and $P \cap\left\{z^{k} \mid k \geq 1\right\}=\varnothing$. Hence, $H \cap P \varsubsetneqq H$ is a closed ideal other than $\{0\}$. This contradicts the definition of $H$.

LEMMA 6. Let $A$ be a commutative Banach algebra such that all prime ideals of $A$ are closed. Then each prime ideal which is not a maximal modular ideal is accessible.

Proof. Let $P \subset A$ be a prime ideal which is not a maximal modular ideal. Then $A / P \neq \mathbb{C}$ is an integral domain of which all prime ideals are closed. By the preceding lemma, $\{0\}$ is accessible in $A / P$; hence, $P$ is accessible in $A$.

THEOREM 4. Let $A$ be a commutative Banach algebra such that all prime ideals of $A$ are closed. Then every derivation on $A$ and every epimorphism onto $A$ has a nilpotent separating space.

Proof. Let $P \subset A$ be a minimal prime ideal. Let $T$ be an epimorphism onto $A$. If $P$ is not a maximal modular ideal, $\mathscr{S}(T)$ is contained in $P$ by the accessibility of the prime ideal. As the separating space of an epimorphism is always contained in the Jacobson radical, $\mathscr{S}(T) \subset P$ holds as well, if $P$ is a maximal modular ideal. Now let $D: A \rightarrow A$ be a derivation. We need only consider the case of $P$ being a maximal modular ideal. Let $\widehat{D}$ denote the derivation on $A / P$ to which $D$ drops according to (1). As the zero-mapping is the only derivation on $A / P=\mathbb{C}$, we have $D A \subset P$, and hence, $\mathscr{S}(D) \subset P$. 
Corollary 2. Let $A$ be a semiprime Banach algebra such that all prime ideals of $A$ are closed. Then every derivation on $A$ and every epimorphism onto $A$ is continuous. In particular, all Banach algebra norms for $A$ are equivalent.

The automatic continuity only of derivations on such Banach algebras has been proved in a different way by Garimella ([4]).

5.2. Topologically simple Banach algebras. Recall the definition of a topologically simple Banach algebra: A Banach algebra $A$ with $A^{2} \neq\{0\}$ is said to be topologically simple if there are no closed, two-sided ideals of $A$ other then $\{0\}$ and $A$. As primitive ideals are automatically closed, a topologically simple Banach algebra is either primitive or radical. It seems to be still unknown, whether topologically simple, radical Banach algebras exist. So, $\mathbb{C}$ is the only known example of a topologically simple, commutative Banach algebra. The question of whether other examples exist is known as the closed ideal problem.

It was Julian Cusack ([2]) who first pointed out the connections between the existence of topologically simple, radical Banach algebras and certain automatic continuity problems for derivations and epimorphisms. In this subsection we use inaccessible prime ideals to give easier proofs of some of his results in the commutative case.

Lemma 7. Let $A \neq \mathbb{C}$ be a commutative Banach algebra which is an integral domain, then the following three statements are equivalent.

(i) $\{0\}$ is inaccessible.

(ii) There is a separating ideal $S \subset A$ other than $\{0\}$.

(iii) There is a separating ideal $S \subset A$ such that $S \cap \operatorname{rad}(A) \neq\{0\}$.

Proof. We only have to show that (i) implies (iii). Let $S$ denote the intersection of all nonzero, closed ideals of $A$, and let $\left\{a_{k}\right\}_{k \geq 1}$ be a sequence in $A$. If there is an $n \in \mathbb{N}$ such that $a_{n}=0$, then

$$
\{0\}=\overline{a_{1} \cdots a_{n} S}=\overline{a_{1} \cdots a_{k} S} \quad(k \geq n) .
$$

Otherwise,

$$
S=\overline{a_{1} S}=\overline{a_{1} \cdots a_{k} S} \quad(k \geq 1) .
$$

Hence, $S$ is a separating ideal other than $\{0\}$, and it is contained in the radical. 
LEMMA 8. Let $A$ be a commutative Banach algebra containing a separating ideal $S$ such that $S \cap \operatorname{rad}(A)$ is not nilpotent. Then $A$ contains an inaccessible prime ideal.

Proof. As $S \cap \operatorname{rad}(A)$ is not nilpotent, there is a minimal prime ideal $P \subset A$ which is not a maximal modular ideal, and which does not contain $S$. By Lemma 2, it is closed, and by Lemma 3, inaccessible.

It is easy to see that a topologically simple, commutative Banach algebra other than $\mathbb{C}$ must be an integral domain; its zero-ideal is obviously inaccessible. On the other hand, if $A$ is a commutative Banach algebra which is an integral domain with inaccessible zero-ideal, then the intersection of all nonzero, closed ideals of $A$ is a topologically simple, commutative Banach algebra which is not isomorphic to $\mathbb{C}$. Further, if $A$ is an arbitrary commutative Banach algebra, and $P \subset A$ is a closed prime ideal, then $P$ is inaccessible in $A$, if and only if the zero-ideal is in $A / P$. Thus, the following assertion holds:

THEOREM 5. The following six statements are equivalent.

(i) There is a commutative Banach algebra $A$ which contains a separating ideal $S$ such that $S \cap \operatorname{rad}(A)$ is not nilpotent.

(ii) There is a semiprime Banach algebra $A$ which contains a separating ideal $S$ such that $S \cap \operatorname{rad}(A) \neq\{0\}$.

(iii) There is a commuutative Banach algebra $A$ which is an integral domain, and which contains a separating ideal $S$ such that $S \cap \operatorname{rad}(A) \neq\{0\}$.

(iv) There is a commutative Banach algebra which is an integral domain with inaccessible zero-ideal.

(v) There is a commutative Banach algebra which contains an inaccessible prime ideal.

(vi) There is a topologically simple, commutative Banach algebra other than $\mathbb{C}$.

It follows easily from Lemma 3 and the above theorem that (b) will be true, if $\mathbb{C}$ turns out to be the only topologically simple, commutative Banach algebra. The next theorem may possibly hint at a way to prove (b) independently of the closed ideal problem.

THEOREM 6. The following four statements are equivalent.

(i) Every derivation on a commutative Banach algebra has a nilpotent separating space.

(ii) Every derivation on a semiprime Banach algebra is continuous. 
(iii) Every derivation on a Banach algebra which is an integral domain is continuous.

(iv) Every derivation on a topologically simple, commutative Banach algebra other than $\mathbb{C}$ is continuous.

Proof. Assume that (iv) holds. Let $A$ be a commutative Banach algebra which is an integral domain, and $D: A \rightarrow A$ a derivation. Without loss of generality we may assume that $\{0\}$ is inaccessible. Let $H$ denote the intersection of all nonzero, closed ideals of $A$, and let $z \in H \backslash\{0\}$. Then $H$ is a topologically simple, commutative Banach algebra other than $\mathbb{C}$, and by (iv), $z D: H \rightarrow H$ is a continuous derivation. Let $\left\{x_{k}\right\}$ be a sequence in $A$ such that

$$
x_{k} \rightarrow 0, \text { and } D x_{k} \rightarrow y .
$$

Then,

$$
z x_{k} \rightarrow 0, \quad \text { and } \quad z D\left(z x_{k}\right)=z^{2} D x_{k}+z(D z) x_{k} \rightarrow z^{2} y .
$$

By the continuity of $z D: H \rightarrow H$, we have $z^{2} y=0$. As $A$ is an integral domain, this implies $y=0$.

Finally, let us add a remark about the prime ideals of a topologically simple, commutative Banach algebra: Given an arbitrary integral domain, we do not know whether nonzero prime ideals exist. In case $A \neq \mathbb{C}$ is a topologically simple, commutative Banach algebra, however, they exist in abundance. In this case, $\bigcap_{k=1}^{\infty} z^{k} A$ is dense in $A$ for any nonzero $z \in A$; furthermore, it does not intersect the multiplicatively closed set $\left\{z^{k} \mid k \geq 1\right\}$. Hence, there is a prime ideal $P \subset A$ with

$$
\{0\} \neq \bigcap_{k=1}^{\infty} z^{k} A \subset P \subset A \backslash\left\{z^{k} \mid k \geq 1\right\} .
$$

Thus, for each nonzero $z \in A$ there is a nonzero prime ideal of $A$ which does not contain $z$.

\section{REFERENCES}

[1] Philip C. Curtis, Derivations on commutative Banach algebras (Proc. Long Beach 1981), Lecture Notes in Math. 975, Springer (1983), 328-333.

[2] J. Cusack, Automatic continuity and topologically simple Banach algebras, J. London Math. Soc. (2), 16 (1977), 493-500.

[3] J. Esterle, Elements for a classification of commutative radical Banach algebras, (Proc. Long Beach 1981), Lecture Notes in Math. 975, Springer (1983), 4-65. 
[4] Ramesh V. Garimella, Continuity of derivations on some semiprime Banach algebras, Proc. Amer. Math. Soc., 99 (1987), 289-292.

[5] B. E. Johnson, Continuity of derivations on commutative Banach algebras, Amer. J. Math., 91 (1969), 1-10.

[6] R. J. Loy, Continuity of derivations on topological algebras of power series, Bull. Austr. Math. Soc., 1 (1969), 419-424.

[7] Uniqueness of the complete norm topology and continuity of derivations on Banach algebras, Tôhoku Math. J., 22 (1970), 471-378.

[8] Volker Runde, Derivationen auf kommutativen Banachalgebren, Schriftenreihe des Mathematischen Instituts der Universität Münster (3), 1 (1990).

[9] A. M. Sinclair, Automatic continuity of linear operators, London Math. Soc., Lecture Notes Series 21, Cambridge Univ. Press, London (1976).

[10] I. M. Singer and J. Wermer, Derivations on commutative normed algebras, Math. Ann., 129 (1955), 260-264.

[11] Marc P. Thomas, The image of a derivation is contained in the radical, Annals of Math., 128 (1988), 435-460.

Received May 1, 1989.

Mathematisches INSTITUT

EINSTEINSTRASSE 62

D-4400 MÜNSTER, W. GeRMANY

Current address: Department of Mathematics

University of California

Berkeley, CA 94720 


\title{
PACIFIC JOURNAL OF MATHEMATICS EDITORS
}

\author{
V. S. VARADARAJAN \\ (Managing Editor) \\ University of California \\ Los Angeles, CA 90024-1555-05 \\ Herbert Clemens \\ University of Utah \\ Salt Lake City, UT 84112 \\ THOMAS ENRIGHT \\ University of California, San Diego \\ La Jolla, CA 92093
}

R. FINN

Stanford University

Stanford, CA 94305

Hermann FlaschKa

University of Arizona

Tucson, AZ 85721

VAUGHaN F. R. JoNes

University of California

Berkeley, CA 94720

Steven Kerckhoff

Stanford University

Stanford, CA 94305

\section{C. MOORE}

University of California

Berkeley, CA 94720

Martin ScharlemanN

University of California

Santa Barbara, CA 93106

HAROLD STARK

University of California, San Diego

La Jolla, CA 92093
R. ARENS
E. F. BECKENBACH
F. WolF
K. YoshidA

\section{ASSOCIATE EDITORS}

\begin{tabular}{ll}
\multicolumn{1}{c}{ SUD-1982) } & \multicolumn{1}{c}{$(1904-1989)$} \\
UNIVERSITY OF ARIZONA & UNIVERSITY OF OREGON \\
UNIVERSITY OF BRITISH COLUMBIA & UNIVERSITY OF SOUTHERN CALIFORNIA \\
CALIFORNIA INSTITUTE OF TECHNOLOGY & STANFORD UNIVERSITY \\
UNIVERSITY OF CALIFORNIA & UNIVERSITY OF HAWAII \\
MONTANA STATE UNIVERSITY & UNIVERSITY OF TOKYO \\
UNIVERSITY OF NEVADA, RENO & UNIVERSITY OF UTAH \\
NEW MEXICO STATE UNIVERSITY & WASHINGTON STATE UNIVERSITY \\
OREGON STATE UNIVERSITY & UNIVERSITY OF WASHINGTON \\
\hline
\end{tabular}

The Supporting Institutions listed above contribute to the cost of publication of this Journal, but they are not owners or publishers and have no responsibility for its content or policies.

\begin{abstract}
Mathematical papers intended for publication in the Pacific Journal of Mathematics should be in typed form or offset-reproduced (not dittoed), double spaced with large margins. Please do not use built up fractions in the text of the manuscript. However, you may use them in the displayed equations. Underline Greek letters in red, German in green, and script in blue. The first paragraph must be capable of being used separately as a synopsis of the entire paper. In particular it should contain no bibliographic references. Please propose a heading for the odd numbered pages of less than 35 characters. Manuscripts, in triplicate, may be sent to any one of the editors. Please classify according to the 1980 Mathematics Subject Classification (1985 Revision) scheme which can be found in the December index volumes of Mathematical Reviews. Supply name and address of author to whom proofs should be sent. All other communications should be addressed to the managing editor, or Elaine Barth, University of California, Los Angeles, California 90024-1555-05.

There are page-charges associated with articles appearing in the Pacific Journal of Mathematics. These charges are expected to be paid by the author's University, Government Agency or Company. If the author or authors do not have access to such Institutional support these charges are waived. Single authors will receive 50 free reprints; joint authors will receive a total of 100 free reprints. Additional copies may be obtained at cost in multiples of 50 .
\end{abstract}

The Pacific Journal of Mathematics (ISSN 0030-8730) is published monthly except for July and August. Regular subscription rate: $\$ 190.00$ a year (10 issues). Special rate: $\$ 95.00$ a year to individual members of supporting institutions.

Subscriptions, orders for numbers issued in the last three calendar years, and changes of address should be sent to Pacific Journal of Mathematics, P.O. Box 969, Carmel Valley, CA 93924, U.S.A. Old back numbers obtainable from Kraus Periodicals Co., Route 100, Millwood, NY 10546.

The Pacific Journal of Mathematics at P.O. Box 969, Carmel Valley, CA 93924 (ISSN 0030-8730) is published monthly except for July and August. Second-class postage paid at Carmel Valley, California 93924, and additional mailing offices. Postmaster: send address changes to Pacific Journal of Mathematics, P.O. Box 969, Carmel Valley, CA 93924.

PUBLISHED BY PACIFIC JOURNAL OF MATHEMATICS, A NON-PROFIT CORPORATION Copyright (C) 1991 by Pacific Journal of Mathematics 